\title{
Paragangliomas of the head and neck region
}

\author{
Paweł Polanowski ${ }^{1}$, Agnieszka Kotecka-Blicharz ${ }^{2}$, Ewa Chmielik ${ }^{3}$, Krzysztof Oleś ${ }^{4}$ \\ Andrzej Wygoda ${ }^{1}$, Tomasz Rutkowski ${ }^{1}$, Marek Kentnowski ${ }^{1}$, Anna Zarudzka ${ }^{1}$, \\ Urszula Dworzecka ${ }^{1}$, Bolesław Pilecki ${ }^{1}$, Kinga Dębiec ${ }^{1}$, Dorota Księżniak-Baran ${ }^{1}$, \\ Joanna Niedziałek ${ }^{1}$, Agata Bieleń ${ }^{1}$, Dominika Leś ${ }^{1}$, Adam Brewczyński ${ }^{1}$, \\ Urszula Wojciechowska ${ }^{5}$, Krzysztof Składowski $^{1}$
}

Paragangliomas of the head and neck are a group of neoplasms which occur very rarely. Most of them are benign tumours. Tinnitus, headaches and dysfunction of the cranial nerves are typical symptoms. Some paragangliomas have metastatic abilities and they can produce catecholamines. There are some typical imaging features in CT and MRI scans which help to determine the correct diagnosis without the necessity of performing a biopsy which can be associated with a haemorrhage risk. Therapeutic management consists of the choice between an active observation, surgical procedure, as well as radiotherapy and systemic therapy in the case of malignant paragangliomas.

NOWOTWORY J Oncol 2018; 68, 3: 132-139

Key words: paraganglioma, radiotherapy, stereotactic radiotherapy

\section{Introduction}

Paragangliomas comprise a group of rarely occurring, richly vascularised, slowly growing, encapsulated neuroendocrine tumours, which develop in various locations of the body, most frequently between the third and sixth decade of life. Taking into consideration the usual benign pathology of paragangliomas, it might seem that they do not pose a serious problem for oncologists specialising in the treatment of malignant tumours of the head and neck region. Such an opinion may also result from the published epidemiological data which specify the incidence of paragangliomas below $0.5 \%$ of all tumours of the head and neck region, which makes up about $0.03 \%$ of all cancers $[1,2]$ as well as from the data from the National Cancer Register: in 2000-2015 only 397 cases of tumours developing from the paraganglial tissue in adrenal and extra-adrenal locations were reported [3]. On the other hand, however, it must be remembered that within the course of the disease, distant metastases might occur; the multifocal character of these tumours paired with their ability to produce catecholamines can lead to a direct threat to patients' lives.

\section{Etiopathogenesis}

These rare tumours develop from paraganglia, which are the accumulations of endocrine cells originating in the neural crest [4]. Paraganglia are dispersed along the autonomic nervous system in the vicinity of sympathetic and parasympathetic neural ganglia or along cranial nerves

${ }^{1} I^{\text {st }}$ Department of Radiotherapy and Chemotherapy, Maria Skłodowska-Curie Institute - Oncology Center, Gliwice Branch, Poland

${ }^{2}$ Department of Nuclear Medicine and Oncological Endocrinology, Maria Skłodowska-Curie Institute — Oncology Center, Gliwice Branch, Poland

${ }^{3}$ Department of Cancer Pathology, Maria Skłodowska-Curie Institute - Oncology Center, Gliwice Branch, Poland

${ }^{4}$ Deepartment of Oncological and Reconstructive Surgery, Maria Skłodowska-Curie Institute - Oncology Center, Gliwice Branch, Poland

${ }^{5}$ Department of Epidemiology and Cancer Prevention, National Cancer Register, Maria Skłodowska-Curie Institute - Oncology Center, Warszawa, Poland 
and large blood vessels. Sympathetic paraganglia have a secretory character and thus secrete catecholamines, whilst parasympathetic paraganglia belong to chemoreceptors and have a receptive character [5]. The division of paragangliomas is presented in Table I. The largest accumulation of paraganglial tissue is located in the adrenal cortex. Tumours originating in this location are defined as pheochromocytomas, and, according to published data, they are the most frequently occurring tumours of the paraganglial system. In the WHO classification, neoplasms developing from extra-adrenal paraganglia are defined as paragangliomas [5, 6]. Paragangliomas of the head and neck region are very rare, making up less than $1 \%$ of all paragangliomas. Their most frequent location in the head and neck region are the following areas: carotid bodies ( $60 \%$ cases), the jugulotympanic area and vagal nerve area (most frequently within a distance of $2 \mathrm{~cm}$ from the skull base) [7].

\section{Nomenclature}

The nomenclature of paragangliomas requires systematisation. The generally operating term, chemodectoma, should in fact refer solely to the tumours of the area of the carotid and aortic bodies, as paraganglia which originate from them, also play the role of chemoreceptors. The most misleading term for paraganglioma, which is frequently used, is glomus tumour. In histology this term refers to the classification of tumours originating from the dermis and subdermal tissue, precisely from the blood vessels, most frequently with a subungual location. Therefore, the use of the term glomus tumour in reference to paraganglioma is an essential error. Recommended terminology, according to the $\mathrm{WHO}$, is meant to refer to paragangliomas with reference to their location, e.g. vagal paraganglioma [7].

In accordance with the classification of head and neck tumours presented by the World Health Organisation (WHO) in 2017, head and neck paragangliomas can be divided into the following:

- carotid body paraganglioma

- laryngeal paraganglioma

- middle ear paraganglioma

— vagal paraganglioma
In comparison with the previous edition of the classification of these tumours, the term jugulo-tympanic paraganglioma was changed into middle ear paraganglioma [8].

\section{The characteristics of paragangliomas}

In the past, paragangliomas were most frequently treated as sporadic, which involved about $90 \%$ of cases [9]. The remaining $10 \%$ were regarded as familial cases. Current studies show that up to $40 \%$ of cases of tumours originating from paraganglial tissues may have a hereditary character [10].

There are 14 various genes which have been identified where germinal mutations can predispose one to the development of pheochromocytomas and paragangliomas. The most frequently identified mutations concern the SDH gene (succinate dehydrogenase complex) and are connected with the familial complex of pheochromocytomas and paragangliomas (PCC/PGL). In the head and neck area this concerns, in particular the D and C subunits (SDHD, SDHC). Gene VHL mutations are also frequent in von Hippel-Lindau syndrome, and also in the RET protooncogene (rearranged during transfection protooncogene) leading to the development of the MEN2 multiple endocrine neoplasia as well as in the NF1 (neurofibromin 1) in the neurofibromatosis type $1[6,11]$.

Paragangliomas usually form isolated lesions, whilst multifocal paragangliomas occur less frequently [12]. Multifocal paragangliomas more often occur among familial ones than in sporadic paragangliomas, which has its justification in the Knudson hypothesis (in sporadic cases, there must be 2 independent mutations in suppressor genes, whilst in hereditary cases, one mutation is congenital and the other - acquired).

Paragangliomas may also be classified with regards to their secretory abilities, into secretory (> 90\%) and non-secretory $(<5 \%)$. The process of secretion is possible due to the presence of main cells, which are the building component of paraganglia. The main cells are the ones which are able to produce catecholamines. Not all secretory paragangliomas produce sufficient amounts of the substance to induce clinical symptoms, that is why some authors define secretory paragangliomas as only those which cause clinical symptoms [13].

Table I. The division of paragangliomas

\begin{tabular}{|c|c|c|}
\hline & Paragangliomas of sympathetic system & Paragangliomas of parasympathetic system \\
\hline Chromium salts staining & Pheochromocytomas & Non-pheochromocytomas \\
\hline Location & Located along sympathetic trunk & Located in the vicinity of the large vessels of head and neck \\
\hline Secretory abilities & Secretory (noradrenaline, adrenaline) & Non-secretory \\
\hline \multirow[t]{2}{*}{ Examples } & $\begin{array}{l}\text { Para-aortic body paragangliomas (ogan of } \\
\text { Zukerkandl) }\end{array}$ & $\begin{array}{l}\text { Carotid body paraganglioma, } \\
\text { jugulo-tympanic paraganglioma, vagal nerve paraganglioma }\end{array}$ \\
\hline & & $\begin{array}{l}\text { Rare locations: paraganglioma of the orbit, oral cavity, larynx, } \\
\text { naso-pharynx, parathyroids }\end{array}$ \\
\hline
\end{tabular}


From a clinical point of view, paragangliomas may be either benign or malignant tumours. Malignant paragangliomas are regarded as only those which are diagnosed after distant metastases have been found in the places where chromogenic tissue does not exist physiologically e.g. to the lymph nodes, bones, lungs or liver [1]. In the current WHO classification, it is assumed that all paragangliomas have metastatic potential. The term_malignant paraganglioma was thus replaced with metastasising paraganglioma or paraganglioma with metastases [8]. Thus the current nomenclature refers to the term "metastasising paraganglioma" instead of "malignant paraganglioma". The prevalence of metastatic paragangliomas is described as ranging between $10-17 \%$. However, this risk strictly depends on the type of germinal mutation underlying the hereditary forms. A mutation in subunit $B$ of succinate dehydrogenase (SDHB) in a hereditary syndrome of pheochromocytomas and paragangliomas may lead to the development of metastases even in $40 \%$ of cases [6].

It must be stressed that $25 \%$ of paragangliomas located in the orbit and larynx are malignant. Also, about $15 \%$ of vagal paragangliomas and $5 \%$ of carotid and jugulotympanic have malignant potential.

Histopathologically, paragangliomas are built out of 2 cell types:

- main cells with excessive eosinophilic cytoplasm and atypical cellular nuclei;

- sustentacular cells of a spindle shape, located on the circumference of the main cell nests.

Tumour cell nests are surrounded with a rich vasculature. The lack of polarisation of the cells on the nest circumferences is a morphological property which allows for the differentiation between paragangliomas and neuroendocrine tumours as this polarisation occurs in the latter The main cells are characterised with the expression of synaptophysin, chromogranin A, CD56 and somatostatin receptors 2A, whilst they do not reveal the expression of cytokeratins, carcino-embryonic antigen (CEA) and calcitonin. Sustentacular cells may be visualised after the application of S-100 or GFAP. No expression is found in these cells, neither there are epithelial or neuroendocrine markers present in them [8]. There are no well-defined and recognised histopathological criteria on the basis of which a metastatic (formerly - malignant) type of paraganglioma can be diagnosed. In some isolated reports, in which in the postoperative histopathological assessment of the necrosis, peri-neural infiltrations, capsular infiltration, increased mitotic activity and atypical mitotic figures, the patients were qualified for postoperative $\mathrm{RTH}$, as the above properties have been regarded as the malignancy criteria [9].

In 2005 Kimura et al., published the scale of the evaluation of pheochromocytomas and extra-adrenal parasympathetic paragangliomas on the basis of the criteria of histological texture, cellularity, coagulative necrosis, vascular invasion and tumour capsule, proliferative index Ki-67 and the types of catecholamines secretion [14]. The application of the above scale allows to classify paragangliomas to one of the three groups: well, moderately or poorly differentiated tumours. The differences between the groups correlate with the metastatic potential and survival rates. Yet, there is a group of well differentiated tumours which still produce metastases, which is indicative of the limitations of the practical application of the Kimura scale [15]. The histopathology of paragangliomas and metastatic tumours is usually the same. Only some metastatic tumours are characterised with a higher proliferative index or lower number of sustentacular cells [15]. The collective analysis of the patients with the diagnosis of paraganglioma or pheochromocytoma, 10-year probability or malignancy is estimated to be about 20\% [16].

\section{Symptoms}

Clinical symptoms presented in the patients with diagnosed paraganglioma are closely connected with the tumour location. The most frequent symptoms are comprised of pulsation in the ears or tinnitus, hearing defects or loss, ear exudate, headaches or cranial nerve dysfunction, comprising: glossopharyngeal nerve (IX), vagal (X), auxiliary (XI), sublingual (XII), in particular in patients with a large tumour mass. Larger tumours in the area of the carotid body are visible in a laryngological assessment in the mid-pharynx or are palpable in the neck, which is the first symptom of paraganglioma, leading to the referral to a specialist and the commencement of a diagnostic proves. Sometimes hoarseness (resulting from the palsy of recurrent laryngeal nerve - branch X) and dysphagia are present.

The main symptom of the jugulotympanic paraganglioma is pulsating tinnitus and conductive hearing defects. In the otoscopic assessment, it is often possible to find a blueish tumour mass shining through the tympanic membrane. Once positive pressure is applied to the tympanic membrane, during the examination with a pneumatic speculum, this mass may go pale. This phenomenon is named Brown's symptom and is indicative of the vascular character of the tumour. Sometimes, paragangliomas are accompanied with atypical clinical symptoms such as excessive sweating (diaphoresis), facial redness, anxiety, vertigo, irregular heart beat and arterial hypertension. These symptoms are the effect of catecholamines secretion, and, in extreme cases may be life threatening. In some isolated cases, the loss of body weight and bone pain may suggest the malignant character of paraganglioma [17].

\section{Imaging diagnostics}

On account of its accessibility, the classical and Doppler ultrasound examinations are used mainly for the lesions 
located in the area of the carotid body - non-homogenous, solid tumours with rich vasculature require extensive diagnostics. The examination which usually suggests a diagnosis of paraganglioma as the first one is the contrast CT.

On account of the rich vascularisation of paragangliomas, the typical CT image is a well delineated hyperdense tumour. CT also allows the visualisation of bone destruction, whose confirmation or exclusion allows to determine the advancement stage of paraganglioma (see Appendix). In the case of a suspected paraganglioma on contrast enhanced CT, MRI must be performed in order to verify it. Upon the administration of a gadolinium contrast agent, paragangliomas reveal strong enhancement. Moreover, the characteristic image of "salt and pepper" in T2-weigthed images allows confirmation of the character of a tumour, which in CT raised a suspicion of paraganglioma [18, 19]. That is why, first of all because of the large risk of a tumour haemorrhage, biopsy is not required in order to confirm diagnosis. The examination necessary for the qualification of patients for paraganglioma resection is angiography which allows visualisation of the network of vessels supplying blood and efferent vessels. The term, "the lyra symptom" encountered in publications, refers to the characteristic image of paraganglioma in angiography, when internal and external carotid arteries are pushed away from each other by the tumour mass developing between them. In the diagnostics of paragangliomas, some other imaging methods, belonging to the domain of nuclear medicine are used as well, comprising: positron emission tomography ( ${ }^{68} \mathrm{Ga}$-DOTATATE, ${ }^{18}$ F-FDG, ${ }^{18}$ F-DOPA tracers) and MIBG 131 -I scintigraphy (iodine-labelled metaiodobenzylguanidine), also with the use of the labelled somatostatin analogues. An indication to perform such examinations is the diagnosis of multi-focal paragangliomas and familial paragangliomas (in particular with the presence of the SDH mutation) in order to exclude distant metastases [16].

\section{Laboratory diagnostics with the suspicion of secretory paragangliomas}

In all patients in whose cases paragangliomas were diagnosed on the basis of imaging diagnostics, it is necessary to perform biochemical diagnostic work comprising the determination of the excretion of fractionated methoxy-catecholamines (metanephrine, normetanephrine, methoxytyramine) in 24-hour urine sampling or free methoxy-catecholamines in serum, depending on the possibilities of the centre in charge; chromogranin A level should be assessed as well [6]. The catecholamines metabolism and metabolites are presented in Figure 1.

\section{Treatment}

The treatment of paragangliomas is a multidisciplinary task. The choice of therapeutic procedures depends on the tumour location and measurements, the patient age, comorbidities, secretory function and the patient's decision. The most appropriate final criterion, used for the analyses comparing the effectiveness of specific methods is local control (LC) and preservation of the nerve function. The overall survival (OS) is here of minor importance with respect to the benign character of the majority of lesions [1].

The following methods of treatment are used:

- active observation;

- surgical resection;

- embolization;

- radiotherapy;

- systemic treatment;

- pharmacotherapy.

\section{CATECHOLAMINES}

\section{METABOLISM PRODUCTS}

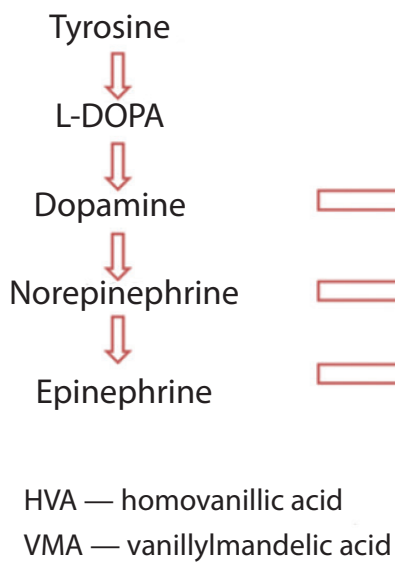

Figure 1. Catecholamines metabolism (on the basis of [13]) 


\section{Active observation}

This method of treatment is recommended especially in elderly patients with an asymptomatic disease, with significant comorbidities. The justification for an active observation is the slow tumour growth (0.5-5 mm annually) and the time of tumour doubling time of between 6 months to 21 years $[20,21]$.

\section{Surgical resection}

Surgery is possible in the majority of paraganglioma locations (especially in the case of cervical paragangliomas with a size below $5 \mathrm{~cm}$ ). Tumour resection is the treatment of choice in patients with metastatic paragangliomas (tumorectomy with cervical lymphadenectomy) or with secretory paragangliomas (in the comparison of the tumour resection with RTH alone, tumour stabilisation was achieved after irradiation with some minor effect regarding the inhibition of secretion). Rich vascularisation and head and neck location areas burden such surgery with a risk of neural and vascular damage as well as with the loss of baroreceptors reflex; this is especially true with bilateral resection of cervical paragangliomas, which is connected with the deregulation of arterial blood pressure. In the case of vagal paragangliomas, the reported rate of nerve $X$ damage is almost $100 \%$ [7]. The risk of death in patients with cervical and vagal paragangliomas undergoing surgery is $1.6 \%$ [22].

The contraindications for surgical resection comprise:

- skull base tumours (difficulties in obtaining complete tumour resection)

- bilateral head and neck paragangliomas

- multifocal paragangliomas

- tumours $>5 \mathrm{~cm}$ (require the reconstruction of cervical vasculature)

\section{Embolisation}

The administration of a vasoconstrictor is rarely used as an independent treatment method. The most frequently used embolization is done in combination with tumour resection which should be at best performed within 48 hours of the application of the embolising agent (polyvinyl alcohol, tris-acryl gelatine microspheres, ethanol, platinum spirals) in order to avoid inducing collateral circulation [23]. Embolisation was for the first time described and recommended by Robertson in 1972. The time span between embolisation and the decision concerning consecutive treatment, including surgical intervention, depends on the resolution or decrease of the symptoms existing before embolisation; it is also necessary to perform follow-up examinations, including an angio-CT [24]. The advantages of this course of treatment comprise the decrease of the tumour mass, which improves resectability conditions and also the decrease of the vascular flow or even a complete occlusion of the vessels, leading to the reduction of the risk of bleeding during the procedure.
It must be remembered, however, that in the case of the displacement of the occlusion material, the risk of ischaemic brain stroke increases.

\section{Radiotherapy}

Irradiation is a generally recognised method of treating paragangliomas, the result of which is the loss of their cell division capability, the creation of vascular emboli and a consecutive vascular fibrosis. Currently, radiotherapy is regarded as the best method of paraganglioma treatment, irrespective of the evaluation of the tumour resectability. The objective of this type of treatment is not complete tumour regression, but the inhibition of its growth. The efficiency of RTH evaluated in follow-up imaging examinations is defined as the lack of tumour progression or its partial regression (partial regression in $61 \%$ patients - a reduction of the baseline tumour dimension by $23 \%$ on average). In comparison with surgery, radiotherapy allows the preservation of the neural function and is connected with a significantly lower risk of vascular complications. It is the method of choice in patients with a diagnosed skull base paraganglioma and vagal nerve paraganglioma. The applied radiotherapy methods are comprised of the standard 3D external beam radiotherapy (EBRT), stereotactic radiotherapy (SBRT) and radiosurgery (SRS). The conventionally fractionated EBRT is usually applied irrespective of the tumour size, up to a total dose of $45 \mathrm{~Gy}$ for 5 weeks [25]. There are reports suggesting that the recurrence rate increases above $1 \%$ when the total irradiation dose is below $40 \mathrm{~Gy}$ [26]. The techniques of stereotactic radiotherapy administered in a few fractions (usually 3-5) up to a total dose of 21-25 Gy or radiosurgery (12-32 Gy administered in a single dose) are applied in particular in the case of tumours which are not larger than $3 \mathrm{~cm}$. Fractionating depends also on the tumour volume. There is also evidence of a higher risk of neuropathy after stereotactic treatment in comparison with EBRT. Better results were observed after a single dose of $15 \mathrm{~Gy}$ in comparison with a single dose of $13 \mathrm{~Gy}$. It was proven that the radiotherapy results are independent of the radiotherapy technique which has been applied (EBRT vs SBRT vs SRS). However, an important aspect is the qualification criterion for each of these methods, which is the tumour size [1]. It was also proven that surgery performed after RTH does not improve the treatment results [27]. In secretory paragangliomas, in spite of increasing EBRT doses to 64-70 Gy, no satisfactory effect inhibiting secretion is achieved; as a result, in such cases the method of choice is tumour resection with a potential postoperative RTH after the consideration of the histopathology results. American researchers performed a metanalysis of paraganglioma treatment methods, comprising: complete resection, partial resection, radiosurgery and partial resection in combination with radiosurgery. The best results with respect to disease recurrence were 
obtained with the application of radiosurgery as an independent method [28]. The generally accessible data from many publications point to a 5 -year and 10-year local disease control after the application of radiotherapy in $99 \%$ and $96 \%$ of patients respectively which shows that irradiation results are at least comparable or better than surgery [29]. On the basis of the American register, National Cancer Data, 5 -year survival totals $60 \%$ in the cases of paragangliomas metastasising to regional lymph nodes [7]. Radiotherapy is also connected with some adverse effects such as mucositis, xerostomia, nausea, progressive hearing loss leading to deafness, osteonecrosis, soft tissues fibrosis, brain necrosis, chronic otitis, otorrhea, nerve VI, VII, VIII palsy — after the administration of doses > 64 Gy, trismus (lockjaw), and also secondary cancers (sarcomas) [21, 30-32]. Mortality connected with conventional radiotherapy is estimated to be about $2 \%$. No deaths related with radiosurgery have been reported [22]. According to Cummings, the treatment results after radiotherapy, depending on the symptoms, were as follows: in 79\% patients tinnitus was reduced, 30\% of patients reported hearing improvement and $5 \%$ complete hearing recovery. $62 \%$ of patients do not experience neither improvement nor deterioration in hearing [33]. The improvement of the function of remaining cranial nerves concerns about $30 \%$ patients [1].

\section{Systemic treatment}

In the cases of diagnosing malignant paragangliomas, which, as was mentioned here, concern patients with metastases, systemic treatment is the basic therapeutic option. The treatment should be conducted by experienced teams consisting of specialists in endocrinology, nuclear medicine and clinical oncology. For the treatment, radionuclides are applied, such as ${ }^{131}$ I-MIBG (in the case of adequate uptake confirmed in scintigraphy: ${ }^{123}$ I- or ${ }^{131}$ I-MIBG), and also isotope-labelled somatostatin analogues (in the cases of confirming the presence of somatostatin receptors: $99 \mathrm{mTc}$ Tectrotid or $\mathrm{PET}^{68} \mathrm{Ga}$-Dotatate). Fast progression of the lesion may be an indication for the use of chemotherapy based on the CVD regimen (cyclophosphamide, vincristine, dacarbazine). Also etoposide, cisplatin and cytosine arabinoses are applied. There are also isolated reports about the efficiency of temozolomide. There are clinical trials in which the targeted treatment with the use of axitinib, pazopanib or sunitinib is studied [16].

\section{Pharmacotherapy}

Pharmacological therapy has not used in a causative treatment although it is frequently applied in the cases of secretory paragangliomas especially before a planned surgery. The medication of first choice comprises selective alpha-1-blockers as these drugs control arterial pressure and prevent catecholaminergic crisis and tachycardia during surgery. In the case of a lack of satisfactory haemodynamic control, it is also possible to include calcium channel blockers into the therapy. Beta-blockers may be used in order to control tachycardia as late as 2-3 days after the use of an alpha-1-blocker. If the drugs inhibiting the beta receptors are introduced sooner there is a risk of developing hypertensive shock [6, 34].

\section{Conclusions}

Paragangliomas, in spite of their very rare incidence, with respect to their biological properties, present themselves as a heterogenous group of cancers. The symptoms of disease are strictly connected with the location of the tumour and the characteristic image observed in CT and MRI is sufficient to make a diagnosis without the necessity of a cytological or histopathological assessment. The therapeutic pattern provides some options for choice between observation and on-going check-ups, surgery and conservative treatment. The most significant criteria determining the treatment method are tumour location, size and hormonal activity. Comparable results after tumour resection and radiotherapy conclude that the choice of treatment is determined by the number of possible complications after the application of each of the methods. Subsequently, radiotherapy may be regarded as the treatment of choice in the treatment of head and neck paragangliomas.

\section{Conflict of interest: none declared}

\section{Paweł Polanowski, MD, PhD}

Maria Skłodowska-Curie Institute _-Oncology Center

Ist Department of Radiotherapy and Chemotherapy

ul. Wybrzeże Armii Krajowej 15

44-101 Gliwice, Poland

e-mail:polanowskipawel@gmail.com

Received: 4 Jun 2018

Accepted: 25 Jul 2018

\section{References}

1. Hu K, Persky MS. Treatment of head and neck paragangliomas. Cancer Control 2016; 23: 228-241.

2. Śniatowska M, Waliszewska-Prosół $M$, Koszewicz $M$ et al. Przyzwojak przebiegający z objawami niedystonicznego kręczu karku. Pol Przegl Neurol 2015; 11: 178-182.

3. Baza Krajowego Rejestru Nowotworów.

4. Sawicki W. Histologia. Warszawa: Wydawnictwo Lekarskie PZWL, 2003: 377-379.

5. Endokrynologia kliniczna: praca zbiorowa. Milewicz A (ed.). Wrocław: Polskie Wydawnictwo Endokrynologiczne, 2012: 424-445.

6. Lenders J, Duh QY, Eisenhofer $\mathrm{G}$ et al. Pheochromocytoma and paraganglioma: an Endocrine Society Clinical Practice Guideline. J Clin Endocrinol Metab 2014; 99: 1915-1942.

7. Bernier J. Head and neck cancer: multimodality management. New York; Springer, 2011: 569-579.

8. El-Naggar AK, Chan JKC, Grandis JR et al. (eds.). WHO classifications head and neck tumors. Lyon: IARC, 2017: 276-284.

9. Kiziltan HS, Ozucer B, Eris AH et al. Bilateral carotid paraganglioma: surgery and radiotherapy. Clin Med Insights Case Rep 2014; 7: 53-57. doi: 10.4137/CCRep.S14223. eCollection 2014

10. Pillai S, Gopalan V, Smith RA et al. Updates on the genetics and the clinical impacts on phaeochromocytoma and paraganglioma in the new era. Crit Rev Oncol Hematol 2016; 100: 190-208. 
11. Baysal BE, Willett-Brozick JE, Lawrence EC et al. Prevalence of SDHB, SDHC, and SDHD germline mutations in clinic patients with head and neck paragangliomas. J Med Genet 2002; 39: 178-183.

12. Myssiorek D. Head and neck paragangliomas: an overview. Otolaryngol Clin North Am 2001; 34: 829-836.

13. Castrucci WA, Chiang VL, Hulinsky I et al. Biochemical and clinical responses after treatment of a catecholamine-secreting glomus jugulare tumor with gamma knife radiosurgery. Head Neck 2010; 32: 1720-1727.

14. Kimura N, Watanabe T, Noshiro T et al. Histological grading of adrenal and extra-adrenal pheochromocytomas and relationship to prognosis: a clinicopathological analysis of 116 adrenal pheochromocytomas and 30 extra-adrenal sympathetic paragangliomas including 38 malignant tumors. Endocr Pathol 2005; 16: 23-32.

15. Mete O, Asa SL (eds.). Endocrine pathology. Cambridge: Cambridge University Press, 2016

16. Angelousi A, Kassi E, Zografos G etal. Metastatic pheochromocytoma and paraganglioma. Eur J Clin Invest 2015; 45: 986-997.

17. Parenti G, Zampetti B, Rapizzi E et al. Updated and new perspectives on diagnosis, prognosis, and therapy of malignant pheochromocytoma/paraganglioma. J Clin Oncol 2012; 2012: 872713. doi: 10.1155/2012/872713.

18. Hu K, Persky MS. Multidisciplinary management of paragangliomas of the head and neck, Part 1.Oncology (Williston Park) 2003; 17: 983-993.

19. Brink I, Hoegerle S, Klisch J et al. Imaging of pheochromocytoma and paraganglioma. Fam Cancer 2005; 4: 61-68.

20. Kataria T, Bisht SS, Mitra S et al. Synchronous malignant vagal paraganglioma with contralateral carotid body paraganglioma treated by radiation therapy. Rare Tumors 2010; 2: e21.

21. Farr HW. Carrotid body tumors. A thirty year experience at Memorial Hospital. Am J Surg 1967; 114: 614-619.

22. Suárez C, Rodrigo JP, Bödeker CC et al. Jugular and vagal paragangliomas: Systematic study of management with surgery and radiotherapy. Head Neck 2013; 35: 1195-1204.

23. Andrews JC, Valavanis A, Fisch U. Management of the internal carotid artery in surgery of the skull base. Laryngoscope 1989; 99: 1224-1229.

24. Lund VJ, Howard DJ, Wei WI et al. Tumors of the nose, sinuses and nasopharynx.Stuttgart; New York: Thieme, 2014.

25. Evenson LJ, Mendenhall WM, Parsons JT et al. Radiotherapy in the management of chemodectomas of the carotid body and glomus vagale. Head Neck 1998; 20: 609-613.
26. Mendenhall WM, Parsons JT, Stringer SP et al. Radiotherapy in the management of temporal bone chemodectoma. Skull Base Surg 1995; 5: 83-91.

27. Kim JA, Elkon D, Lim ML et al. Optimum dose of radiotherapy for chemodectomas of the middle ear. Int J Radiat Oncol Biol Phys 1980; 6: 815-819.

28. Gottfried ON, Liu JK, Couldwell WT. Comparison of radiosurgery and conventional surgery for the treatment of glomus jugulare tumors. Neurosurg Focus 2004; 7: E4.

29. Gilbo P, Tariq A, Morris CG et al. External-beam radiation therapy for malignant paraganglioma of the head and neck. Am J Otolaryngol 2015; 36: 692-696.

30. Powell S, Peters N, Harmer C. Chemodectoma of the head and neck: results of treatment in 84 patients. Int J Radiat Oncol Biol Phys 1992; 22: 919-924.

31. Dawes PJ, Filippou M, Welch AR et al. The management of glomus jugulare tumours. Clin Otolaryngol Allied Sci 1987; 12: 15-24.

32. Lybeert ML, van Andel JG, Eijkenboom WM et al. Radiotherapy of paragangliomas. Clin Otolaryngol Allied Sci 1984; 9: 105-109.

33. Cummings BJ, Beale FA, Garrett PG et al. The treatment of glomus tumors in the temporal bone by megavoltage radiation. Cancer 1984; 53: $2635-2640$.

34. Interna Szczeklika 2015. Gajewski P (ed.). Kraków: Wydawca Medycyna Praktyczna, 2015: 1352-1357.

35. Trombetta M, Silverman J, Colonias A et al. Paraganglioma: a potentially challenging tumor. Oncology (Willston Park) 2008; 22: 341-343.

36. Jackson CG, Harris PF, Glasscock ME $3^{\text {rd }}$ et al. Diagnosis and management of paragangliomas of the skull base. Am J Surg1990; 159: 389-393.

37. Fisch U. Mattox DE. Microsurgery of the skull base. Stuttgart; New York: Thieme-Verlag, 1988: 149-152.

38. Capatina C, Ntali G, Karavitaki N et al. The management of head-and-neck paragangliomas. Endocr Relat Cancer 2013; 20: R291-R305.

39. Shamblin WR, ReMine WH, Sheps SG et al. Carotid body tumor (chemodectoma). Clinicopathologic analysis of ninety cases. Am J Surg 1971; 22: 732-739.

40. Luna-Ortiz K, Rascon-Ortiz M, Villavicencio-Valencia V et al. Does Shamblin's classification predict postoperative morbidity in carotid body tumors? A proposal to modify Shamblin's classification. Eur Arch Otorhinolaryngol 2006; 263: 171-175. [Errata in: Eur Arch Otorhinolaryngol 2006; 263: 1161].

41. Ng E, Duncan G, Choong AM et al. Sclerosing paragangliomas of the carotid body: A series of a rare variant and review of the literature. Ann Vasc Surg 2015; 29: 1454.e5-1454.e12. 


\section{Appendix}

\section{Glasscock-Jackson Classification}

\section{Cervical body paragangliomas [35]}

- I - small tumour covering the area of the jugular vein bulb, middle ear and mastoid process

- II - tumour growing below internal acoustic meatus intracranial expansion possible

- III - tumour expanding to the top of the petrous pyramid of the temporal bone - intracranial expansion is possible

- IV - the tumour expanding beyond the top of the petrous pyramid of the temporal bone to the clivus or subtemporal fossa - intracranial expansion is possible

\section{Tympanic paragangliomas [36]}

- Period I - small tumour in the area of promontorium

- Period II - the tumour fills the tympanic cavity

- Period III - the tumour fills the tympanic cavity, penetrating into the mastoid process

- Period IV - the tumour fills the tympanic cavity, mastoid process, external acoustic meatus and may infiltrate towards internal carotid artery

\section{Fisch and Mattox classification [37, 38]}

The classification of jugulo-tympanic paragangliomas (former nomenclature)

- stage A - tumour develops from tympanic plexus

- stage B - tumour develops is hypotympanum, infiltrates middle ear and mastoid process

- stage C1 - tumour destroys the foramen of the carotid artery without infiltrating carotid artery

- stage C2 - tumour destroys the vertical part of the carotid artery canal

- stage C3 - tumour destroys the horizontal part of the carotid artery canal, without infiltrating foramen lacerum

- stage C4 - tumour infiltrates foramen lacerum and cavernous sinus

- stage De1/2 - tumour proliferates intracranially, but epidurally: De1 not more than $2 \mathrm{~cm}$, De2 - more than $2 \mathrm{~cm}$;

- stage Di1/2/3 - tumour proliferates intracranially and in intradural manner: Di1 do $2 \mathrm{~cm}$; Di2 - between 2 and $4 \mathrm{~cm}$; Di3 - more than $4 \mathrm{~cm}$.

Table II. Shamblin's classification of paragangliomas [39]

\begin{tabular}{llll}
\hline Stage & Tumour & $\begin{array}{l}\text { Difficulty degree of } \\
\text { tumour resectability }\end{array}$ & Surgery \\
\hline I & The tumour separates ICA and ECA, attaches to the bifurcation & Low & Routine dissection \\
II & Partly covers the vessels & Medium & Subintimal dissection \\
III & Closely covers the vessels & High & Partial or complete vascular resection \\
\hline
\end{tabular}

Table III. Shamblin's modified classification of paragangliomas [40, 41]

\begin{tabular}{llll}
\hline Stage & Tumour size & $\begin{array}{l}\text { Cervical vessel involvement or infiltration in the tumour } \\
\text { area }\end{array}$ & $\begin{array}{l}\text { Difficulty degree of tumour resectability } \\
\text { II }\end{array}$ \\
\hline IIIA & $>4 \mathrm{~cm}$ & $\begin{array}{l}\text { The tumour does not involve or infiltrate cervical vessels } \\
\text { Partially involves cervical vessels }\end{array}$ & $\begin{array}{l}\text { No difficulties } \\
\text { Difficult }\end{array}$ \\
IIIB & Closely involves cervical vessels & $\begin{array}{l}\text { Difficult, requires vessel correction, removal, or } \\
\text { replacement }\end{array}$ \\
& Each & $\begin{array}{l}\text { Stage I, II or III in Shamblin's original classification with } \\
\text { the infiltration of cervical vessels }\end{array}$ & $\begin{array}{l}\text { The clinical and/or histopathological confirmation of } \\
\text { the vascular wall invasion is necessary }\end{array}$ \\
\hline
\end{tabular}

